\title{
Rate of concept identification and the noticeability of the relevant dimension'
}

\author{
KEITH N. CLAYTON, COLEMAN T. \\ $M E R R Y M A N, 2$ and THOMAS $B$. \\ LEONARD, III, 3 Vanderbilt University, \\ Nashville, Tenn. 37203
}

Six groups of Ss were given concept identification tasks involving stimuli varying in six dimensions. $A$ different dimension was relevant for each of the six groups and the observed identification rate was compared to measures of relative salience of the dimensions provided by a separate set of Ss. The failure to obtain a positive correlation between the measure of relative salience of a dimension and ease of concept identification when that dimension was relevant is discussed in terms of a distinction between "noticing" a cue and "using" a cue.

The importance of perceptual processes in studies of concept identification has perhaps been most explicitly recognized by Bower \& Trabasso (1964). Indeed, they have argued that a theory of concept identification must "imply that the rate of learning should vary directly with the. . .salience of the relevant cues [Trabasso \& Bower, 1968, p. 44]." To represent the salience of a cue, Trabasso and Bower assigned to it a weight, $w_{i}$, which is assumed to "summarize the effects of all those factors that influence the noticeability or attention value of a cue [p. 51]." When the cue is placed in the context of a total of $\mathrm{N}$ cues, the relative weight of Cue $i, a_{i}$, is defined as

$$
\text { (1) } a_{i}=w_{i} / \sum_{j=1}^{N} w_{j} \text {. }
$$

Since $a_{i}$ is assumed to be the equivalent of the probability of selecting Cue $\mathrm{i}$ from the population of $\mathbf{N}$ cues, $a_{i}$ becomes the learning-rate parameter and $\mathrm{Eq} .1$ gives the relation between cue salience and learning rate when Cue $i$ is the only relevant cue.

Previous research, which has been reviewed by Trabasso \& Bower (1968), has shown that concept identification can be facilitated if the values of the relevant dimension are "emphasized" or made dissimilar. For example, using flower patterns as stimuli, Trabasso (1963) found that identification of the relevant dimension, stem-leaves angle, was faster when the angle was colored red than when the color of the angle was the same as the rest of the flower. Similarly, Archer (1962) found concept identification to be faster when the difference between the two values of the relevant dimension was large than when the difference was small. These two operations, increasing "emphasis" and "obviousness," are assumed to increase the salience of the affected dimension. In the present study a different technique was used to evaluate the contribution of cue salience to concept identification. First, a large number of $S s$ were presented with stimuli varying in six dimensions. Each $S$ was asked to describe how the stimuli differed and to indicate the order in which the differences became apparent to him. These rankings provided the basis for a measure of relative salience of the six dimensions. Then, a different set of Ss was given a concept-identification task involving the same stimuli. These Ss were divided into six groups. For each group only one dimension was relevant, and a different dimension was relevant for each of the six groups. Thus, an attempt was made to measure the relative salience of all of the dimensions and then to determine whether rate of concept identification was related to the measured salience of the relevant dimension.

\section{MEASURES OF SALIENCE Stimulus Materials}

The stimuli were geometric forms varying in six dimensions: color (red or blue), shape (square or circle), number (one or two figures), size (large or small), position (located on the left or right half of the stimulus field), and brightness (dark or light). Since each dimension was binary there were exactly $2^{6}=64$ different stimuli. Two sets of these stimuli were used in this experiment, the first was constructed by placing the forms on white $5 \times 8$-in. cards, the second by taking $35-\mathrm{mm}$ slides of the original set.

\section{Procedure}

Each $S$ was shown a subset of the stimuli after having been informed that the stimuli would "differ in a number of ways." The S's task was to describe the differences which were noticed in the order in which the differences became apparent to him.

This stage of the research was carried out in three separate experiments with slightly differing procedures. For example, in two of the experiments two stimuli (slides) were presented successively with the second member of the pair differing in all six dimensions from the first. In the other experiment the pair of stimuli (cards) were presented simultaneously, one on top of the other. Further, in two of the experiments the Ss were run individually and asked to verbalize the order in which the dimensions were noticed. In the other experiment the $S s$ were run in a single group and asked to write down their responses. Since the results obtained seemed to be unaffected by these procedural variations, the results presented here are the combined results.

Subjects

The Ss for the measures of salience were 46 male and 60 female students in an introductory course of psychology who participated as part of the course requirement.

\section{CONCEPT IDENTIFICATION TASK}

Stimulus Materials and Subjects

The stimuli were the same as the $35-\mathrm{mm}$ slides described above. They were presented by a Carousel projector onto a screen approximately $30 \mathrm{ft}$ in front of the S. The Ss ( 60 males and 60 females) were drawn from the same pool as described above but were not the same $S$ s as those who participated in the measures of salience.

\section{Procedure}

Each $S$ was run individually and instructed that he was to classify each stimulus by responding " $A$ " or " $B$ " after which the $\mathrm{E}$ would tell him the correct response. The procedures of the task followed closely those used by Bower \& Trabasso (1964) except that before beginning each $S$ was informed what the six dimensions were and told the correct classification depended upon only one of the six dimensions. There were six groups of $20 \mathrm{Ss}, 10 \mathrm{male}$ and 10 female, and a different dimension was relevant for each of the six groups. Within each subgroup half of the Ss had response " $A$ " paired with a particular attribute on the relevant dimension. Each $S$ was run to a criterion of 15 consecutive correct responses.

$$
\text { RESULTS }
$$

\section{Measures of Salience ${ }^{4}$}

Three scores are reported in Table 1 for each dimension. The first column gives the number of Ss reporting to have noticed the dimension first. A $\chi^{2}$ test on these data permits a rejection of the hypothesis that each dimension is equally likely to be noticed first $\left(\chi^{2}=99.13, \mathrm{df}=5, \mathrm{p}<.001\right)$. The average rank-order of noticeability is presented in the second column of Table 1. Overall, the measures of relative salience of the six dimensions seem to fall into three groups. First, color and shape were noticed early and shape was noticed first about as often as was color. Brightness, on the other hand, was almost always the last dimension noticed as indicated by its mean rank of 5.68 
Table 1

Measure of Salience of Each Dimension and Number of Errors in Concept Identification Task

\begin{tabular}{lccr}
\hline & \multicolumn{2}{c}{ Measure of Salience } & $\begin{array}{c}\text { Concept Identification } \\
\text { Task }\end{array}$ \\
\hline & $\begin{array}{c}\text { Number of Ss Noticing } \\
\text { First }\end{array}$ & Mean Rank Order & Mean Errors \\
\hline Color & 43 & 2.14 & \\
Shape & 39 & 2.05 & 7.40 \\
Number & 14 & 3.46 & 8.05 \\
Position & 7 & 3.81 & 10.25 \\
Size & 3 & 3.73 & 3.90 \\
Brightness & 0 & 5.68 & 8.20 \\
\hline
\end{tabular}

out of a possible 6 . The other three dimensions, size, position, and number, were essentially equally intermediate between these two extremes.

Concept Identification Task

The mean number of errors-to-criterion by each of the six groups is given in the third column of Table 1 . There it can be seen that concept identification was easiest when position or brightness was relevant and most difficult when number was relevant. A test of the differences among the six groups was provided by an extension of a likelihoodratio test (Bower \& Trabasso, 1964,Eq. S3, p. 60) on the estimates of the learning-rate parameters of the six groups. The obtained ratio, 15.74 , should be distributed under the null hypothesis as $\chi^{2}$ with $\mathrm{df}=5$. Therefore, the null hypothesis of no differences among the learning-rate parameters of the six groups may be rejected with $\mathrm{p}<.01$. Comparisons between adjacent pairs of groups confirm the impression provided in Table 1, namely position and brightness were significantly easier than the other four, but no significant differences were obtained else where.

Turning now to a comparison of the measure of salience and concept-identification rate, it is clear from Table 1 that the two measures are poorly correlated. For example, brightness was one of the last dimensions reported to be noticed, yet concept identification was relatively easy when brightness was relevant. Indeed, a rank-order correlation between the mean rank order on the measure of noticeability and the mean errors in the concept identification task, although nonsignificant, is negative (rho $=-.49$ ). DISCUSSION

In the present experiment no support was provided for the prediction that dimensions which ranked high in their noticeability would be relatively easy dimensions to identify in the conceptidentification task. Though there are several possible explanations of this finding, only one will be discussed in this report. The key to the explanation lies in a recent distinction made by Trabasso \& Bower (1968) between "noticing" a cue and "using" the cue. As noted in the introduction, the prediction of a positive correlation between cue salience and concept-identification rate depends upon the assumption that if a dimension is noticed, it will be used. Some recent work by Downing (in press) provides some evidence that this assumption is incorrect. Downing used the Levine (1966) technique for tracking Ss' hypotheses in combination with a postexperimental interview. As in the present study, Downing obtained no correlation between concept-identification rate and a measure of dimension salience (the time required to note a difference on the dimension). Reports Downing: "Only $16 \%$ of the Ss did not judge color to be the most obvious, yet $61 \%$ of the Ss did not test color first. Among the Ss who did not test color first, $77 \%$ said that color was too obvious and therefore did not think it would be the relevant cue."

The distinction between noticing and using a cue should also alleviate the apparent contradiction between the results of the present study and those of Suchman \& Trabasso (1966) who obtained a positive correlation between measures of children's preference for dimensions and rate of discrimination performance. It is quite likely that the Suchman-Trabasso preference data provide a measure of the tendency to use a cue whereas the present measures of cue salience merely reflect the probability of noticing a cue. In any case, the results of the present study, along with those of Downing suggest that the relative noticeability of dimensions contributes only indirectly to concept identification performance.

\section{REFERENCES}

ARCHER, E. J. Concept identification as a function of obviousness of relevant and irrelevant information. Joumal of Experimental Psychology, 1962, 63, 616-620.

BOWER, G. H., \& TRABASSO, R. T. Concept identification. In R. C. Atkinson (Ed.), Studies in mathematical psychology. Stanford, California: Stan ford University Press, 1964. Pp. 32-94.

DOWNING, B. D. Discriminability and preference in concept identification. Psychonomic Science, in press.

LEVINE, M. Hypothesis behavior by humans during discrimination learning. Journal of Experimental Psychology, 1966, 71, 331-338.

SUCHMAN, R. G., \& TRABASSO, T. R. Stimulus preference and cue function in young children's concept attainment. Journal of Experimental Child Psy chology, 1966, 3, 188-198.

TRABASSO, T. R. Stimulus emphasis and all-or-none learning in concept identification. Journal of Experimental Psychology, 1963,65, 398-406.

TRABASSO, T. R., \& BOWER, G. H. Attention in learning: Theory and research. New York: J, Wiley \& Sons, Inc., 1968. NOTES

1. A portion of this paper was presented at the annual convention of the Midwestern Psychological Association, 1965.

2. Now at Indiana University, Bloomington, Indiana 47401.

3. Now at the University of Mississippi, University, Mississippi 38677.

4. Significant sex differences were obtained from the cue-salience measures. However, since the conclusions drawn in the present report hold regardless of sex, the differences will be ignored here. A description of the sex differences is available from the first au thor upon request. 